\title{
Impact of Flow Rate, Collection Devices, and Extraction Methods on Tear Concentrations Following Oral Administration of Doxycycline in Dogs and Cats
}

\author{
Lionel Sebbag,, ${ }^{1,2}$ Lucas Showman, $^{3}$ Emily M. McDowell, Ann Perera, and Jonathan P. Mochel ${ }^{1}$
}

\begin{abstract}
Purpose: Compare the precision of doxycycline quantification in tear fluid collected with either Schirmer strips or polyvinyl acetal (PVA) sponges following oral drug administration.

Methods: Three dogs and 3 cats were administered doxycycline orally at a dose of $4.2-5 \mathrm{mg} / \mathrm{kg}$ every $12 \mathrm{~h}$ for 6 consecutive days. At day 5 and 6, blood and tear fluid were sampled to capture doxycycline trough and maximal concentrations. Tear fluid was collected 3 times (spaced $10 \mathrm{~min}$ apart) at each session with the absorbent material placed in the lower conjunctival fornix until the 20-mm mark was reached (Schirmer strip, one eye) or for $1 \mathrm{~min}$ (PVA sponge, other eye). Tear extraction was performed with either centrifugation or elution in methanol. Doxycycline concentrations were measured with liquid chromatography-mass spectrometry. Low $(100 \mathrm{ng} / \mathrm{mL})$ and high $(1,000 \mathrm{ng} / \mathrm{mL})$ tear concentrations measured in vivo were spiked into each absorbent material in vitro to evaluate percentage drug recovery. Results: After oral administration of doxycycline, the drug reached the tear compartment at concentrations of $45.1-900.7 \mathrm{ng} / \mathrm{mL}$ in cats and $45.4-632.0 \mathrm{ng} / \mathrm{mL}$ in dogs, representing a tear-to-serum ratio of $12 \%$ and $16 \%$, respectively. Doxycycline tear concentrations were significantly more precise when tear collection was performed with Schirmer strips rather than PVA sponges $(P=0.007)$, but were not correlated with tear flow rate. In vitro doxycycline recovery was poor to moderate $(<75 \%)$.

Conclusions: Schirmer strips represent a good option for lacrimal doxycycline quantification, although the collection and subsequent extraction have to be optimized to improve drug recovery.
\end{abstract}

Keywords: pharmacokinetics, doxycycline, oral administration, tear film, canine, feline

\section{Introduction}

$\mathbf{S}$ YSTEMICALly ADMINISTERED MEDICATIONS are used to manage a variety of ocular surface diseases across species, for instance oral cetirizine for allergic conjunctivitis in humans, ${ }^{1}$ famciclovir for herpetic keratitis in cats, ${ }^{2}$ and doxycycline for keratomalacia in horses, ${ }^{3}$ among others. Although promising, the prerequisite for such therapies is a deep understanding of the drug pharmacokinetics-pharmacodynamics at the ocular surface, ${ }^{4}$ a process that first requires accurate quantification of the drug levels in the tear fluid. Indeed, under- or overestimating tear concentrations could cause a drug to be given at an inappropriate dose and/or frequency, thereby affecting its efficacy, increasing the risk for drug toxicity, or development of acquired resistance (for anti-infective drugs). ${ }^{5,6}$
However, accurate quantification of drug in tears is complicated by the lack of standardized method to collect and analyze this biological fluid. ${ }^{7-10}$ This is exemplified by doxycycline, a broad-spectrum antibiotic that has been shown to reach the tear compartment in various species: After oral administration, doxycycline tear concentrations were relatively high in horses $(1,810-9,830 \mathrm{ng} / \mathrm{mL}),{ }^{11}$ lower in cats $(100-110 \mathrm{ng} / \mathrm{mL}),{ }^{12}$ much lower in dogs $(0.04-5.73 \mathrm{ng} / \mathrm{mL}),{ }^{13}$ and nonexistent in humans. ${ }^{14}$ The subtle species differences in plasma protein binding of doxycycline ${ }^{15}$ cannot solely explain the large discrepancies in the observed tear concentrations; rather, it is likely that the concentrations obtained have been skewed by an unrecognized impact from the tear collection process, as is the case for tear protein content ${ }^{16}$ and other analytes like glucose, ${ }^{17}$ and warrants further investigation.

Departments of ${ }^{1}$ Biomedical Sciences and ${ }^{2}$ Veterinary Clinical Sciences, Iowa State University, College of Veterinary Medicine, Ames, Iowa.

${ }^{3}$ W.M. Keck Metabolomics Research Laboratory, Iowa State University, Ames, Iowa.

${ }^{4}$ Lloyd Veterinary Medical Center, Iowa State University, College of Veterinary Medicine, Ames, Iowa.

(C) Lionel Sebbag et al. 2018; Published by Mary Ann Liebert, Inc. This Open Access article is distributed under the terms of the Creative Commons License (http://creativecommons.org/licenses/by/4.0), which permits unrestricted use, distribution, and reproduction in any medium, provided the original work is properly cited. 
Direct tear collection with capillary tubes, although commonly used in clinical research because the sample obtained is readily available for analysis, ${ }^{8}$ presents several drawbacks that limit its use for drug quantification. In particular, the volume of tear fluid retrieved is generally low, thus rendering some samples insufficient for analysis ${ }^{1}$ unless previous stimulation or topical instillation of saline is performed. ${ }^{18}$ In addition, the drug recovery and the accuracy of analysis are lower than other methods, as shown by Small et al. who noted that capillary-collected samples underestimated ofloxacin concentrations in rabbit tears and resulted in greater variability when compared with Schirmer strips and surgical sponges. ${ }^{19}$

Indirect tear collection with Schirmer strips and ophthalmic sponges have been successfully used to quantify lacrimal drug levels in several species. ${ }^{2,13,19,20}$ These collection methods are easy to use, safe, and provide quantities of tear fluid adequate for most bioanalytical procedures. ${ }^{7,21,22}$ However, Schirmer strips and ophthalmic sponges can also cause reflex tearing due to ocular irritation and therefore affect the detection of the investigated tear component (eg, drug concentration). ${ }^{21,22}$ Standardization of the collection methods and controlling tear flow rate may hinder these physiological changes and improve the repeatability of the analysis.

The purpose of our study is to compare the repeatability (ie, precision) of drug quantification on the ocular surface from tears collected with Schirmer strips or ophthalmic sponges. We also aim to investigate the impact of the method used to extract the tears from the absorbent materials, that is, centrifugation or elution with a solvent. Doxycycline was selected as the test drug because it has been shown to reach the tear compartment in several species, ${ }^{11-13}$ it has broad-spectrum antimicrobial activity, and multiple beneficial properties such as inhibition of matrix metalloproteinases, anti-inflammation, immunomodulation, and antiangiogenesis. ${ }^{23}$

\section{Methods}

\section{In vivo evaluation: determination of serum and tear levels}

Animals. Three dogs and 3 cats were enrolled in the study; all confirmed to be ophthalmoscopically healthy by slit-lamp examination, indirect funduscopy, tonometry, Schirmer tear test, and fluorescein staining. Dogs comprised of 1 neutered male Chihuahua, 1 spayed female Great Pyrenees, and 1 spayed female Greyhound, with a mean \pm standard deviation (SD) (range) age of $5.7 \pm 3.5$ years ( $2-9$ years) and body weight of $19.3 \pm 13.0 \mathrm{~kg}(6-32 \mathrm{~kg})$. Cats were all Domestic Shorthair breed, comprised of 2 neutered male and 1 spayed female, with a mean $\pm \mathrm{SD}$ (range) age of $3.7 \pm 2.3$ years (1-5 years) and body weight of $5.8 \pm 1.4 \mathrm{~kg}(5-7.5 \mathrm{~kg})$. An informed consent was obtained from all owners. The study was approved by the Institutional Animal Care and Use Committee of Iowa State University, and adhered to the Association for Research in Vision and Ophthalmology (ARVO) statement for the Use of Animals in Ophthalmic and Vision Research.

Drug administration and sample collection. Doxycycline hyclate was administered orally at a dose of $4.2-5 \mathrm{mg} / \mathrm{kg}$ every $12 \mathrm{~h}$ for 6 consecutive days. On both the fifth and sixth day of drug administration, all subjects had blood and tear fluid sampled to capture doxycycline trough concentration $\left(C_{\text {trough }}\right.$; before morning drug administration) and maximal concentration $\left(C_{\max } ; 3 \mathrm{~h}\right.$ after morning drug administration), as described in Fig. 1. The duration of drug administration preceding the first sample collection was selected to ensure steady-state doxycycline plasma concentrations were reached, as the plasma half-life was reported to be $10.36 \mathrm{~h}$ in $\operatorname{dogs}^{24}$ and $4.24 \mathrm{~h}$ in cats. ${ }^{12}$

Blood was collected once from each subject at each session ( $C_{\text {trough }}$ and $C_{\max }$ at day 5 and 6): the $2-3 \mathrm{~mL}$ blood sample retrieved by peripheral venipuncture was placed in a plain red top tube (containing no anticoagulant), centrifuged at $1,240 \mathrm{~g}$ for $10 \mathrm{~min}$, and the extracted serum was stored at $-80^{\circ} \mathrm{C}$ until analysis.

Tear fluid was collected 3 times from each subject at each session ( $C_{\text {trough }}$ and $C_{\max }$ at day 5 and 6), allowing $10 \mathrm{~min}$ between successive sampling in the same eye to permit return to basal tearing status. ${ }^{22}$ For each subject, the right and left eyes were randomized (Excel 2016; Microsoft, Redmond, WA) to receive either Schirmer strips (Eye Care Product Manufacturing, LLC, Tucson, AZ) or polyvinyl acetal (PVA) sponges (Ultracell ${ }^{\circledR}$ Eye Wick; Beaver-Visitec International, Inc., Waltham, MA), and this selection was kept consistent over the several sessions. Collection with Schirmer strips was performed by placing the filter paper in the lower conjunctival fornix until the strip was wet at the 20-mm mark. Collection with PVA sponges was performed as previously described ${ }^{22}$ briefly, a $4 \times 10 \mathrm{~mm}$ strip of PVA sponge was inserted into the lower conjunctival fornix using a disposable plastic tweezer (Evident Inc., Union Hall, VA), and was left in place for $60 \mathrm{~s}$. Once soaked with tears, extraction of tear fluid from these absorbent materials was performed using 1 of 2 methods:

(1) Centrifugation: The wetted Schirmer strips and PVA sponges were placed in separate 0.2-mL Eppendorf tubes that were previously punctured at their bottom with an 18-gauge needle. Tears were then eluted through the drainage hole into $1.5-\mathrm{mL}$ Eppendorf tubes by centrifugation for $1 \mathrm{~min}$ at $3,884 \mathrm{~g}\left(\mathrm{VWR}^{\circledR}\right.$ Mini Centrifuge; VWR International, Radnor, PA).

(2) Methanol elution: The wetted Schirmer strips and PVA sponges were placed in separate 1.5 -mL Eppendorf tubes. Methanol (HPLC grade; Fisher Scientific, Raleigh, NC) was then added to each tube to immerse the entire absorbent material $(1.5 \mathrm{~mL}$ for Schirmer strips, $1 \mathrm{~mL}$ for PVA sponges).

During each time point, 2 of the 3 tear samples collected from each eye were processed using the same extraction method (centrifugation on day 5, methanol elution on day 6; Fig. 1) so that intrasession variability could be assessed (see "Data Analysis" section).

Assuming tear fluid density equaled $1 \mathrm{~g} / \mathrm{mL}^{25}$ tear volume absorbed was calculated as the difference of the post- and precollection weight of the tube containing the Schirmer strip or PVA sponge (before centrifugation or methanol elution). Weights were determined to the nearest $0.001 \mathrm{~g}$ (Gemini-20; American Weight Scales, Inc., Norcross, GA). Samples were then placed in $\mathrm{a}-80^{\circ} \mathrm{C}$ freezer until analysis.

\section{In vitro evaluation: determination of doxycycline recovery from absorbent materials}

Doxycycline hyclate (Sigma-Aldrich $\geq 98 \%$ HPLC; SigmaAldrich, St Louis, MO) was prepared as solutions of 100 and $1,000 \mathrm{ng} / \mathrm{mL}$ (representative of low and high tear concentrations 


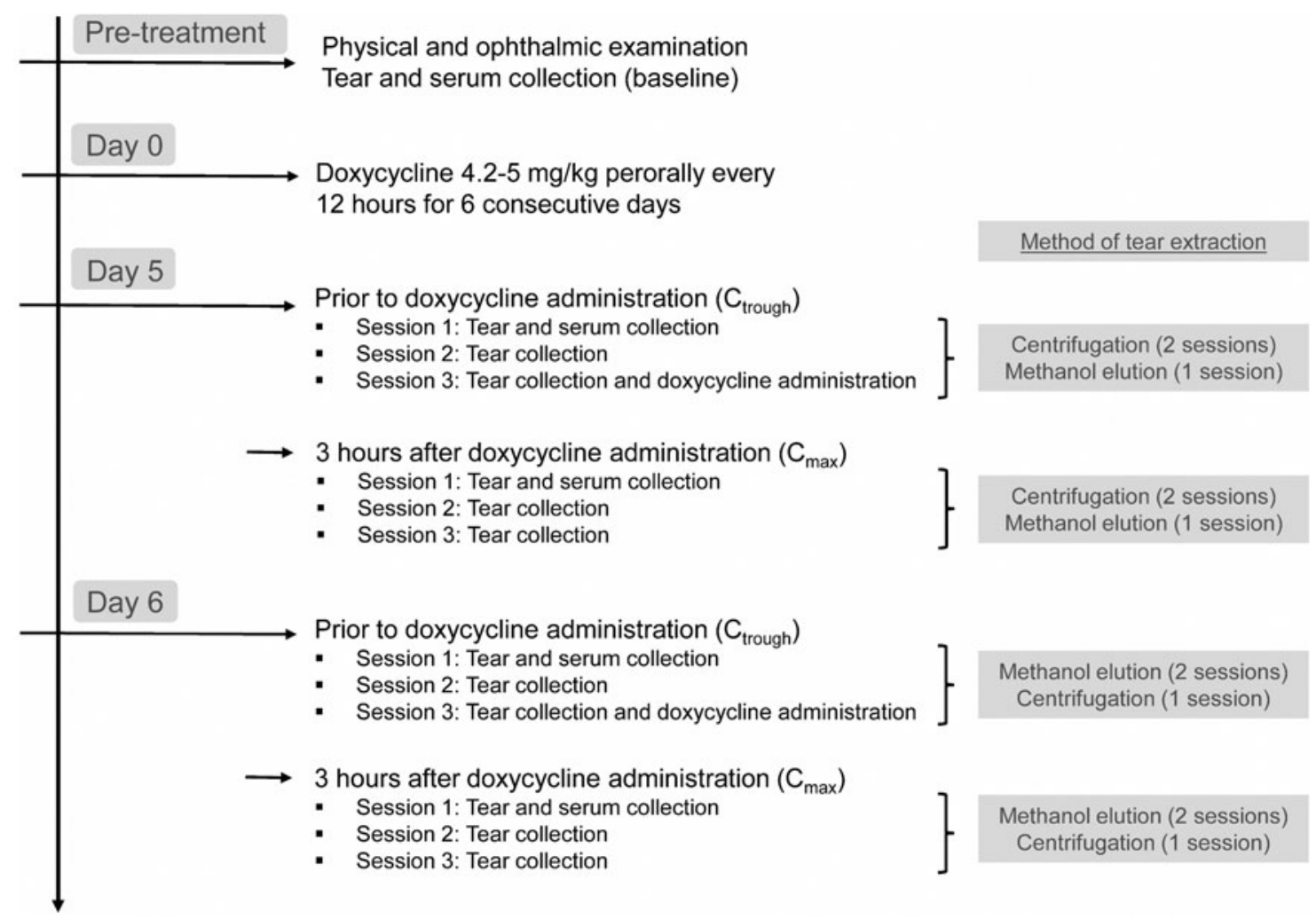

FIG. 1. Diagram of the study design used to assess 3 healthy dogs and 3 healthy cats who were administered oral doxycycline $(4.2-5.0 \mathrm{mg} / \mathrm{kg}$ every $12 \mathrm{~h}$ for 6 days). At both day 5 and 6 , serum and tear samples were collected to capture trough $\left(C_{\text {trough }}\right)$ and maximal $\left(C_{\max }\right)$ concentrations. Tears were collected 3 times at each session, separated 10 min apart, and fluid was extracted from the absorbent material with either centrifugation or methanol elution.

obtained in the present in vivo experiment) in a buffer made of $50 \%$ acetonitrile (HPLC grade; Fisher Scientific, Raleigh, NC), $50 \%$ water (HPLC grade; Fisher Scientific, Raleigh, NC), and $0.1 \%$ formic acid. Twenty Schirmer strips (wet until the $20-\mathrm{mm}$ mark was reached) and 20 PVA sponges (spiked with $25 \mu \mathrm{L}$, representative of the median volume absorbed by these sponges in the present in vivo experiment) were used for each doxycycline concentration, 10 of which underwent extraction with centrifugation while the remaining 10 underwent elution with methanol, following the same protocol as the in vivo experiment. Samples were then stored at $-80^{\circ} \mathrm{C}$ until analysis.

\section{Analytical methods}

Sample preparation. Serum and tear samples were thawed and vortexed gently for 1 min (Vortexer 120V; Fisher Scientific, Hampton, NH). Tear samples obtained by methanol elution were centrifuged for $6 \mathrm{~min}$ at $10,000 \mathrm{~g}$ (Labnet International, Edison, NJ), and the supernatants ( $0.5 \mathrm{~mL}$ for PVA samples and $1.0 \mathrm{~mL}$ for Schirmer samples) were transferred to LC-MS vials. The samples were dried with a nitrogen evaporator for $20 \mathrm{~min}$, then resuspended in $200 \mu \mathrm{L}$ of buffer (1:1 acetonitrile:water with $0.1 \%$ formic acid). For tears obtained by centrifugation and for serum samples, $7-10$ and $100 \mu \mathrm{L}$ of each sample type, respectively, were transferred to $1.5-\mathrm{mL}$ microcentrifuge tubes and mixed with 200 or $900 \mu \mathrm{L}$ of buffer (1:1 acetonitrile:water with $0.1 \%$ formic acid), respectively. These samples were then vortexed for $30 \mathrm{~s}$, left on ice for $10 \mathrm{~min}$, and vortexed for another $30 \mathrm{~s}$ before being sonicated in an ice water bath for
$10 \mathrm{~min}$. Following centrifugation for $7 \mathrm{~min}$ at $13,000 \mathrm{~g}$, the supernatant from each sample was transferred to a $0.2 \mu \mathrm{L}$ spin filter and centrifuged again for $7 \mathrm{~min}$ at $13,000 \mathrm{~g}$.

Standard solutions. The following concentrations of doxycycline hyclate were prepared in a buffer of 1:1 acetonitrile:water with $0.1 \%$ formic acid: $1.0,5.0,10,20,50$, $100,625,1,000,1,250,2,500$, and 5,000 ng/mL. Tear calibration curve was made by analyzing doxycycline solutions of $1,5,10,20,50$, and $100 \mathrm{ng} / \mathrm{mL}$ that were each mixed with $10 \mu \mathrm{L}$ of blank canine and feline tears. Serum calibration curve was made by analyzing doxycycline solutions of 625 , $1,250,2,500$, and $5,000 \mathrm{ng} / \mathrm{mL}$ that were each mixed with $30 \mu \mathrm{L}$ of blank canine and feline serum. A linear correlation was observed within the range of concentrations considered for tears $\left(R^{2}=0.989\right)$ and for serum $\left(R^{2}=0.996\right)$.

Liquid chromatography/mass spectrometry. An Agilent 6540 Ultra High-Definition Accurate-Mass Q-TOF liquid chromatography/mass spectrometry (LC/MS) system was used for sample analysis (Agilent Technologies, San Jose, CA). LC separations were performed with an Agilent Technologies' 1290 Infinity Binary Pump UHPLC instrument equipped with an Agilent Technologies' Eclipse C18 $1.8 \mu \mathrm{m} 2.1 \mathrm{~mm} \times 50 \mathrm{~mm}$ analytical column that was coupled to an Agilent Technologies' 6540 UHD Accurate-Mass QTOF mass spectrometer. A volume of $15 \mu \mathrm{L}$ of each sample was injected into the device, with an initial buffer condition of $100 \%$ water progressing to $100 \%$ acetonitrile with $0.1 \%$ formic acid over the 10-min run period. Chromatography 
was carried out at $40^{\circ} \mathrm{C}$ with a flow rate of $0.6 \mathrm{~mL} / \mathrm{min}$. Doxycycline was detected as $[\mathrm{M}+\mathrm{H}]^{+}$ions using electrospray ionization in positive mode, while operating in highresolution $(4 \mathrm{Gz})$ mode with a scan range from $\mathrm{m} / \mathrm{z} 100$ to $\mathrm{m} / \mathrm{z} 1,700$. Doxycycline $[\mathrm{M}+\mathrm{H}]^{+}$ions were plotted as an extracted ion chromatogram using a $10 \mathrm{ppm}$ extraction window centered around $\mathrm{m} / \mathrm{z} 445.16$, and the observed retention time for doxycycline was $3 \mathrm{~min}$.

Analyses of blank tear fluid spiked with 2 doxycycline standard solution (100 and 1,000 ng/mL) were performed to serve as control for the in vitro study. For the in vivo experiments, the concentrations of doxycycline in the tear fluid and serum were estimated in $\mathrm{ng} / \mathrm{mL}$ on the basis of peak area from the tears and serum calibration curves, respectively, taking into account the various dilutions performed during the sample preparation.

\section{Data analysis}

Normality of the data was assessed with the Shapiro-Wilk test. For normally distributed data, parametric tests were used for analysis and results were expressed as mean \pm SD. Nonnormally distributed data were analyzed with nonparametric statistics and results were expressed as median and 95\% range (2.5th percentile-97.5th percentile). Comparison of serum doxycycline levels was performed with the unpaired $t$-test (dogs vs. cats) and the Mann-Whitney rank test $\left(C_{\text {trough }}\right.$ vs. $\left.C_{\max }\right)$. Comparison of tear doxycycline levels between dogs versus cats, and between $C_{\text {trough }}$ versus $C_{\max }$ were performed with the Kruskal-Wallis test followed by Dunn's pairwise comparison, taking into account the following 4 collection/extraction methods: (1) Schirmer strip/centrifugation, (2) Schirmer strip/ methanol elution, (3) PVA sponge/centrifugation, and (4) PVA sponge/methanol elution. When no statistical differences were noted, data from both species as well as both trough and maximal concentrations were combined for future analysis, described thereafter as "serum doxycycline concentration" and "tear doxycycline concentration." Differences in the doxycycline concentrations obtained in vivo and in vitro with each of the aforementioned group were evaluated with the KruskalWallis test followed by Dunn's pairwise comparison.

Correlations. Spearman's rank correlation tests were used to assess correlations between the following parameters: (1) tear doxycycline concentration versus tear flow rate (defined as the volume absorbed by the absorbent material per minute), and (2) serum doxycycline concentration versus tear doxycycline concentration obtained by each of the 4 collection/extraction methods.

Intrasession variability. The variability of the tear doxycycline concentrations obtained by the same method during the same session was evaluated with the coefficient of variation (CV), expressed in \%, defined as the ratio of the SD by the mean values of doxycycline concentrations. Differences in CV\% between Schirmer strips and PVA sponges were evaluated with the Mann-Whitney test, whereas differences in CV\% among the 4 collection/extraction methods were assessed with the Kruskal-Wallis test.

\section{Results}

Data were non-normally distributed $(P<0.05)$, except for serum doxycycline concentrations $(P \geq 0.354)$ and tear volume absorbed $(P \geq 0.118)$.

\section{In vivo evaluation: determination of serum and tear levels}

Serum concentrations of doxycycline were not statistically different between trough and maximal measurements in both species $(P \geq 0.650)$. The drug could be quantified in all sera samples collected: mean $\pm \mathrm{SD}$ (range) concentrations were $1301.8 \pm 602.0 \mathrm{ng} / \mathrm{mL}(469.1-2356.5 \mathrm{ng} / \mathrm{mL})$ in dogs and $1531.6 \pm 804.6 \mathrm{ng} / \mathrm{mL}(329.5-3208.4 \mathrm{ng} / \mathrm{mL})$ in cats, a difference that was not statistically significant $(P=0.437)$. Mean \pm $\mathrm{SD}$ (range, $\mathrm{CV} \%$ ) tear volume absorbed was $17.6 \pm 2.3 \mu \mathrm{L}$ (13-22 $\mu \mathrm{L}, 13 \%)$ for Schirmer strips and $27.6 \pm 17.7 \mu \mathrm{L}(3-$ $70 \mu \mathrm{L} ; 64 \%$ ) for PVA sponges. Tear concentrations of doxycycline were below the limit of quantification $(<1 \mathrm{ng} / \mathrm{mL})$ in 29/144 samples collected, a finding mostly noted in cats and methanol-eluted tears (Appendix 1). Median (95\% range) tear levels were $182.0 \mathrm{ng} / \mathrm{mL}(45.4-632.0 \mathrm{ng} / \mathrm{mL})$ in dogs and $172.0 \mathrm{ng} / \mathrm{mL}(45.1-900.7 \mathrm{ng} / \mathrm{mL})$ in cats, a difference that was not statistically significant $(P=0.865)$.

Tear concentrations were not statistically different between trough and maximal concentrations in both species and in both collection methods $(P=1.000$; Table 1$)$. In tear samples collected with Schirmer strips, centrifugation extraction achieved greater doxycycline levels than methanol elution for both $C_{\text {trough }}(P=0.002)$ and $C_{\max }(P<0.001$; Table 1$)$. In tear samples collected with PVA sponges, doxycycline levels were greater with centrifugation extraction for $C_{\max }(P=0.025)$, but not $C_{\text {trough }}(P=0.121 ;$ Table 1$)$.

Table 1. Median \pm Standard Error (95\% Range) Trough and Maximal Concentrations of Doxycycline in Tear Fluid of 3 Healthy Dogs and 3 Healthy Cats Receiving 4.2-5 Mg/Kg Doxycycline Per orally for 6 Consecutive Days

\begin{tabular}{|c|c|c|c|c|}
\hline \multirow{2}{*}{$\begin{array}{l}\text { In vivo } \\
\text { Doxycycline } \\
\text { concentration }\end{array}$} & \multicolumn{2}{|c|}{ Schirmer strips } & \multicolumn{2}{|c|}{ PVA sponges } \\
\hline & Centrifugation & Methanol elution & Centrifugation & Methanol elution \\
\hline $\begin{array}{l}C_{\text {trough }} \\
(\mathrm{ng} / \mathrm{mL})\end{array}$ & $279.6 \pm 24.2(143.3-458.2)^{*}$ & $63 \pm 57.9(44.8-543.7)$ & $176 \pm 59.2(119.3-831.8)$ & $65.4 \pm 12.7(33.7-138.8)$ \\
\hline$C_{(\mathrm{max}}$ & $441.1 \pm 79.2(308.8-1388.1)^{*}$ & $87 \pm 53.9(53.9-156.6)$ & $278 \pm 26.1(186.4-552.5)^{*}$ & $96.8 \pm 57.2(38.7-650.7)$ \\
\hline
\end{tabular}

Tears were collected in vivo with either Schirmer strips or PVA sponges, and fluid was subsequently extracted by centrifugation or methanol elution. For the same absorbent material, significant differences between centrifugation versus methanol are noted with an asterisk $(*)$.

PVA, polyvinyl acetal. 
Correlations. Concentrations of doxycycline were not correlated between serum and tear fluid for any collection/ extraction method $(P \geq 0.221)$, except for Schirmer strips followed by centrifugation $(r=0.424, P=0.013)$. Furthermore, no significant correlation was noted between tear flow rate and tear doxycycline concentrations for either Schirmer strips $(P=0.87$; Fig. 2 A $)$ or PVA sponges $(P=0.640$; Fig. $2 \mathrm{~B})$.

Intrasession variability. Median \pm standard error $(95 \%$ range) $\mathrm{CV} \%$ of tear samples collected with Schirmer strips $(9.7 \% \pm 3.4 \%, 1.1 \%-46.6 \%)$ was significantly lower $(P=0.007)$ compared with samples collected with PVA sponges $(21.4 \% \pm 7.6 \%$; 6.7\%-93.0\%; Fig. 3). When considering the subsequent extraction method, CV\% was confirmed to be lower for Schirmer strips [centrifugation: $8.5 \% \pm 5.0 \%$ (0.8\%-47.6\%); methanol elution: $14.3 \% \pm 3.8 \%(2.8 \%-25.3 \%)]$ compared with PVA sponges [centrifugation: $21.4 \% \pm 8.7 \%$ (8.5\%-92.5\%); methanol elution: $34.2 \% \pm 17.6 \% \quad(8.0 \%-$ $76.3 \%)$, although differences among these 4 groups were not statistically significant $(P \geq 0.054)$.

\section{In vitro evaluation: determination of doxycycline recovery from absorbent materials}

Results of the in vitro experiment are summarized in Table 2. There were no significant differences $(P \geq 0.210)$ in the recovery of doxycycline between Schirmer strips and PVA sponges for either extraction methods (centrifugation or methanol-elution) or doxycycline solutions (100 and $1,000 \mathrm{ng} / \mathrm{mL}$ ). Median CV\% of doxycycline quantitation was overall similar between Schirmer strips and PVA sponges for the $100 \mathrm{ng} / \mathrm{mL}$ solution $(35.7 \%$ and $32.1 \%$, respectively) and the $1,000 \mathrm{ng} / \mathrm{mL}$ solution $(19.4 \%$ and $27.2 \%$, respectively). The recovery of doxycycline from the absorbent materials was often greater with $100 \mathrm{ng} / \mathrm{mL}$ versus $1,000 \mathrm{ng} / \mathrm{mL}$ of doxycycline solution, and this difference was statistically significant for centrifuged PVA sponges $(P<0.001)$.

\section{Discussion}

Precise quantification of tear fluid concentrations following systemic therapy is critical for many reasons. First, systemic



medications can be used to treat various ocular surface diseases, and accurate tear concentrations are necessary to determine dose and frequency of administration. Second, lacrimal fluids can be used to correlate drug concentrations with ocular adverse effects of systemic drugs, such as conjunctivitis from nonsteroidal anti-inflammatories ${ }^{26}$ or druginduced corneal epithelial changes. ${ }^{27}$ Third, tears have been shown to be the best practical indicator of the unbound fraction of various drugs, such as phenobarbital ${ }^{28}$ and theophylline, ${ }^{29}$ and therefore represent an excellent alternative to invasive blood or cerebrospinal fluid collection.

After oral administration, doxycycline reached the tear compartment at concentrations of $45.1-900.7 \mathrm{ng} / \mathrm{mL}$ in cats and $45.4-632.0 \mathrm{ng} / \mathrm{mL}$ in dogs. In fact, these values may represent underestimations of the "true" concentrations, as our preliminary in vitro data showed an incomplete recovery of doxycycline from the absorbent materials with the extraction protocols used in the present study. Furthermore, the analytical method used in the present study did not use an isotopically labeled internal standard of doxycycline, a limitation that likely affected the accuracy of the measurements from the LC/MS assay. ${ }^{30}$ Thus, the range of tear concentrations in dogs and cats needs to be substantiated by additional studies with a larger population size. Regardless of these limitations, these measured concentrations are up to 9-fold and 1,000-fold greater than those previously reported in cats and dogs, respectively. ${ }^{12,13}$ Because serum doxycycline concentrations were overall consistent with data published in previous canine and feline studies using similar doses of oral doxycycline, ${ }^{12,31}$ the large discrepancy in concentrations measured in the lacrimal fluid could be imputed, at least partially, to the different methodologies used for tear collection and extraction.

The average tear-to-serum ratio of doxycycline concentrations was $16 \%$ in dogs and $12 \%$ in cats, somewhat similar to penciclovir $(20 \%)^{2}$ but lower than pradofloxacin in cats (36$1,818 \%){ }^{12}$ These differences in lacrimal distribution might be due to physicochemical properties inherent to each drug such as lipophilicity, protein binding, molecular mass, and $\mathrm{p} K_{\mathrm{a} .}{ }^{10}$ Of note, the tear-to-serum ratio was greater in tear samples collected with Schirmer strips and extracted with centrifugation $(27 \%)$ compared with other methods $(5 \%-$ $16 \%$ ). This, combined with the steady volumes of tear fluid

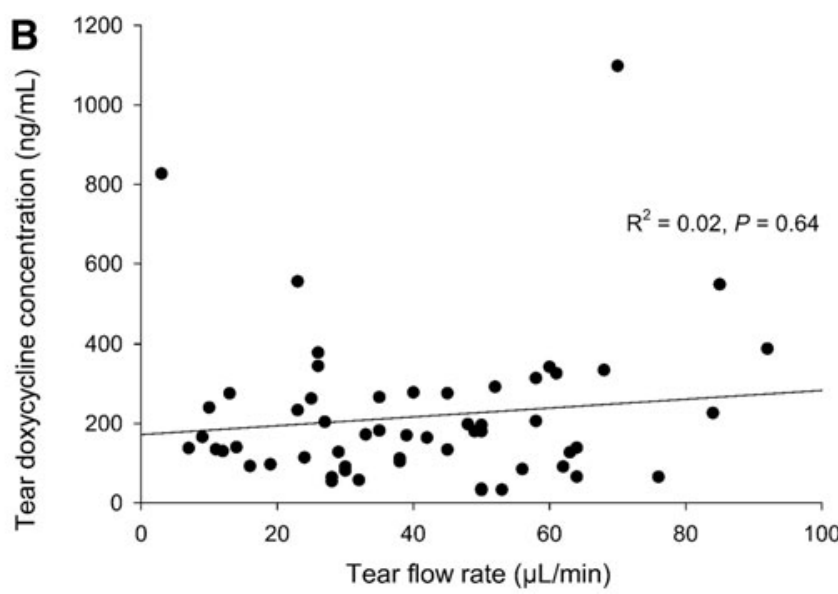

FIG. 2. Spearman's rank correlation test for tear doxycycline concentrations and tear flow rate. No significant associations were found for either Schirmer strips (A) or PVA sponges (B). PVA, polyvinyl acetal. 


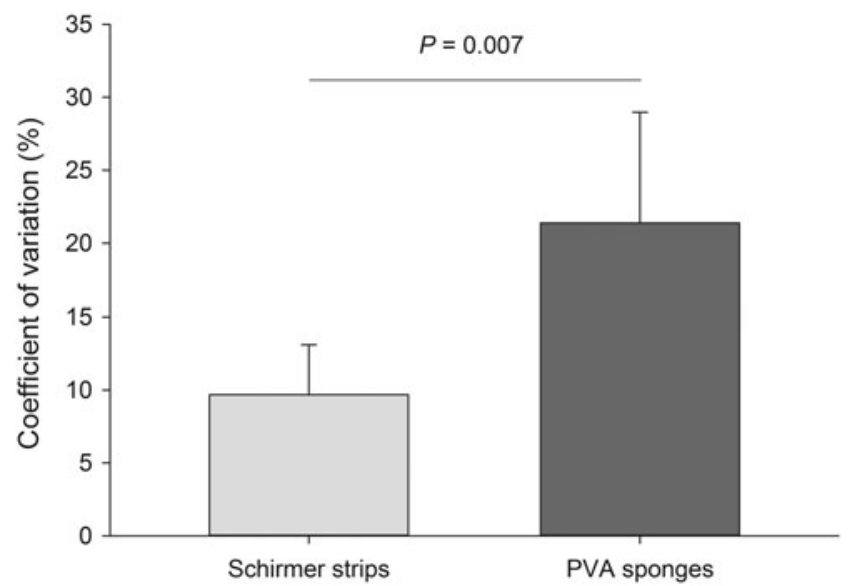

FIG. 3. Bar chart depicting median + standard error of the coefficient of variation in doxycycline concentrations of tears collected in vivo with Schirmer strips or PVA sponges. A significant difference was noted between both collection methods $(P=0.007)$. PVA, polyvinyl acetal.

obtained with Schirmer strips at each collection time (20-mm mark wetting), may partly explain the positive correlation noted for this method between serum and tear concentrations of doxycycline. Furthermore, it is important to note that our work and the aforementioned studies were performed in healthy eyes. Breakdown of the blood-ocular barrier, as seen with conjunctivitis-induced vessel fragility, may increase the tear-to-serum ratio by allowing direct passage of drug from the serum to the tear fluid. ${ }^{1}$ In humans with allergic conjunctivitis, oral administration of cetirizine achieved tear concentrations nearly identical to serum concentrations after $60 \mathrm{~min}$, indicating that a pharmacokinetic equilibrium has been reached relatively quickly between the 2 compartments. ${ }^{1}$ In humans with trachomatous conjunctivitis, administration of minocycline achieved sustained tear concentrations for $48 \mathrm{~h}$ following a single oral dose. ${ }^{32}$ Future studies should investigate the impact of conjunctivitis on lacrimal drug levels.

In the present study, doxycycline tear concentrations were not correlated with tear flow rate in dogs and cats. The stable concentration profile of tear doxycycline over a wide range of tear flow rates suggest that the drug secretion into the tear compartment increases with the rate of lacrimal stimulation. This is similar to "regulated" lacrimal proteins, such as lysozyme and lactoferrin, 33 but is in contrast to "constitutive" and "serum-derived" tear compounds, such as IgA and $\mathrm{IgG}$, respectively, for which the tear concentrations decrease with increasing stimulus due to dilution effect. ${ }^{33}$ Additionally, it is plausible that doxycycline accumulates in the lacrimal gland after days of oral administration, similar to cyclosporine $\mathrm{A},{ }^{18}$ thus explaining the pattern of concentrations observed in the present study.

Importantly, our study found that doxycycline tear concentrations were more repeatable when tear collection was performed with Schirmer strips rather than PVA sponges. Schirmer strips are thinner than PVA sponges $(0.22 \mathrm{~mm}$ vs. $1 \mathrm{~mm})$ and this may help extract the drug from the absorbent material in a more consistent way, thus providing a possible explanation for the lower variability of doxycycline in tear samples. Further, differences in water affinity between Schirmer strips and PVA sponges, ${ }^{22}$ combined with different physical properties, such as porosity, pore size, and permeability, ${ }^{34}$ may explain the (nonsignificant) discrepancy noted herein in the quantity of doxycycline extracted from each collection device.

Of note, Schirmer strips allow for standardization of the tear volume absorbed at each session by controlling the mmmark of wetness (intersession differences in VA $\leq 4 \mu \mathrm{L}$ ), whereas PVA sponges yield more variable tear volumes despite controlling for the collection duration (intersession differences in VA $\leq 37 \mu \mathrm{L}$ ). This variability is likely due to reflex tearing, and although it did not impact doxycycline concentrations in the present study (no correlation between tear flow rate and doxycycline concentrations), it may participate in data variability when tear sampling is not performed at steady-state plasma levels. Indeed, a recent study on minocycline collected tear fluid in ponies with ophthalmic sponges, and the authors noted large discrepancies in lacrimal drug concentrations between one subject to another. ${ }^{35}$ Therefore, we believe that Schirmer strips represent a better option for lacrimal doxycycline quantification, offering a good compromise between collection of a sufficient amount of tears (for allowing bioanalytical quantitation) and patient tolerance (for the respect of lacrimal physiology). However, this finding should be verified for other drugs as results may be affected by diverse compound characteristics (eg, size, lipophilicity, and protein binding). Also, given the several in vivo samples that were below the quantification limit and our preliminary in vitro data showing suboptimal drug recovery, tear collection with Schirmer strips has to be optimized for it to become the standard collection method for pharmacokinetic studies. Indeed, postcollection processing of Schirmer strips has been shown to impact the lacrimal protein content, ${ }^{36}$ and the same is likely to be true for drug levels. The following are a few suggestions that can be investigated in future studies:

Table 2. Median \pm Standard Error (95\% Range) Percentage Recovery of Doxycycline IN Vitro From SCHIRMER STRIPS AND PVA SPONGES SPIKED WITH EITHER 100 OR 1,000 NG/ML Doxycycline Solutions, Then Processed with Either Centrifugation or Methanol Elution

\begin{tabular}{|c|c|c|c|c|}
\hline \multirow{2}{*}{$\begin{array}{l}\text { In vitro } \\
\text { Doxycycline } \\
\text { solution }\end{array}$} & \multicolumn{2}{|c|}{ Schirmer strips } & \multicolumn{2}{|c|}{ PVA sponges } \\
\hline & Centrifugation & Methanol elution & Centrifugation & Methanol elution \\
\hline $100 \mathrm{ng} / \mathrm{mL}$ & $60.7 \pm 13.2(32.1-100.8)$ & $52.3 \pm 3.4(46.3-64.5)$ & $75.0 \pm 1.9(67.4-77.0)^{*}$ & $38.4 \pm 2.0(35.2-44.1)$ \\
\hline $1,000 \mathrm{ng} / \mathrm{mL}$ & $31.8 \pm 3.0(22.6-38.5)$ & $40.2 \pm 1.0(39.4-43.4)$ & $24.8 \pm 1.1(22.9-27.5)$ & $40.0 \pm 2.1(37.1-45.6)$ \\
\hline
\end{tabular}

For the same absorbent material and the same extraction method, significant differences between the recovery from $100 \mathrm{ng} / \mathrm{mL}$ versus $1,000 \mathrm{ng} / \mathrm{mL}$ solutions are noted with an asterisk (*).

PVA, polyvinyl acetal. 
(1) Adjust the volume absorbed by the Schirmer strip to collect only the basal lacrimal lake $(6.2-7.0 \mu \mathrm{L}$ in humans, ${ }^{37}$ unknown in dogs and cats), so that any dilution effect from reflex tearing is reduced. ${ }^{19}$ If so, extraction of the tears with solvent elution would be preferable, as the amount of fluid recovered by centrifugation of such low volumes is often negligible ( $<5 \mu \mathrm{L}$; L. Sebbag, unpublished observations).

(2) Improve the extraction of drug by cutting the Schirmer strip in small pieces, ${ }^{13}$ allowing sufficient contact time between the solvent and the Schirmer strip ${ }^{36}$ and using ultrasonic agitation to facilitate diffusion. ${ }^{12,20}$

(3) Establish the analytical calibration curve by spiking Schirmer strips with known concentrations of the drug of interest, and processing these standards the same way as the biological samples. ${ }^{38}$ This approach may help account for the potential adsorptive properties of the Schirmer strips for a wide range of drug concentrations.

\section{Author Disclosure Statement}

No competing financial interests exist.

\section{References}

1. Grumetto, L., Cennamo, G., Del Prete, A., La Rotonda, M.I., and Barbato, F. Pharmacokinetics of cetirizine in tear fluid after a single oral dose. Clin. Pharmacokinet. 41:525531, 2002.

2. Sebbag, L., Thomasy, S.M., Woodward, A.P., Knych, H.K., and Maggs, D.J. Pharmacokinetic modeling of penciclovir and BRL42359 in the plasma and tears of healthy cats to optimize dosage recommendations for oral administration of famciclovir. Am. J. Vet. Res. 77:833-845, 2016.

3. Ollivier, F.J., Brooks, D.E., Kallberg, M.E., et al. Evaluation of various compounds to inhibit activity of matrix metalloproteinases in the tear film of horses with ulcerative keratitis. Am. J. Vet. Res. 64:1081-1087, 2003.

4. Dave, V.S., and Bhansali, S.G. Pharmacokinetics and pharmacodynamics of ocular drugs. In: Pathak, Y., Sutariya, V., and Hirani, A.A., eds. Nano-Biomaterials for Ophthalmic Drug Delivery. Cham: Springer International Publishing; 2016; p. 111-129.

5. Craig, W.A. Pharmacokinetic/pharmacodynamic parameters: rationale for antibacterial dosing of mice and men. Clin. Infect. Dis. 26:1-10, 1998.

6. Fink, M., Letellier, I., Peyrou, M., Mochel, J.P., Jung, M., King, J.N., Gruet, P., and Giraudel, J.M. Population pharmacokinetic analysis of blood concentrations of robenacoxib in dogs with osteoarthritis. Res. Vet. Sci. 95:580-587, 2013.

7. Zhou, L., and Beuerman, R.W. Tear analysis in ocular surface diseases. Prog. Retin. Eye Res. 31:527-550, 2012.

8. Rentka, A., Koroskenyi, K., Harsfalvi, J., et al. Evaluation of commonly used tear sampling methods and their relevance in subsequent biochemical analysis. Ann. Clin. Biochem. 54:521-529, 2017.

9. Heinig, K., Bertran, E., Potter, J., and Fraier, D. Ocular bioanalysis: challenges and advancements in recent years for these rare matrices. Bioanalysis. 9:1997-2014, 2017.

10. Raju, K.S., Taneja, I., Singh, S.P., and Wahajuddin. Utility of noninvasive biomatrices in pharmacokinetic studies. Biomed. Chromatogr. 27:1354-1366, 2013.
11. Baker, A., Plummer, C.E., Szabo, N.J., Barrie, K.P., and Brooks, D.E. Doxycycline levels in preocular tear film of horses following oral administration. Vet. Ophthalmol. 11: 381-385, 2008.

12. Hartmann, A., Krebber, R., Daube, G., and Hartmann, K. Pharmacokinetics of pradofloxacin and doxycycline in serum, saliva, and tear fluid of cats after oral administration. J. Vet. Pharmacol. Ther. 31:87-94, 2008.

13. Collins, S.P., Labelle, A.L., Dirikolu, L., Li, Z., Mitchell, M.A., and Hamor, R.E. Tear film concentrations of doxycycline following oral administration in ophthalmologically normal dogs. J. Am. Vet. Med. Assoc. 249:508-514, 2016.

14. Smith, V.A., Khan-Lim, D., Anderson, L., Cook, S.D., and Dick, A.D. Does orally administered doxycycline reach the tear film? Br. J. Ophthalmol. 92:856-859, 2008.

15. Riond, J.L., and Riviere, J.E. Doxycycline binding to plasma albumin of several species. J. Vet. Pharmacol. Ther. 12:253-260, 1989.

16. Runstrom, G., Mann, A., and Tighe, B. The fall and rise of tear albumin levels: a multifactorial phenomenon. Ocul. Surf. 11:165-180, 2013.

17. Baca, J.T., Finegold, D.N., and Asher, S.A. Tear glucose analysis for the noninvasive detection and monitoring of diabetes mellitus. Ocul. Surf. 5:280-293, 2007.

18. Mora, P., Ceglarek, U., Manzotti, F., et al. Cyclosporin A in the ocular fluids of uveitis patients following long-term systemic administration. Graefes Arch. Clin. Exp. Ophthalmol. 246:1047-1052, 2008.

19. Small, D., Hevy, J., and Tang-Liu, D. Comparison of tear sampling techniques for pharmacokinetics analysis: ofloxacin concentrations in rabbit tears after sampling with schirmer tear strips, capillary tubes, or surgical sponges. J. Ocul. Pharmacol. Ther. 16:439-446, 2000.

20. Dumortier, G., Zuber, M., Barges, N., Chast, F., Dutertre, H., and Chaumeil, J.C. Lacrimal and plasmatic kinetics of morphine after an ophthalmic delivery of three different formulations. Drug Dev. Ind. Pharm. 20:1147-1158, 1994.

21. Dumortier, G., and Chaumeil, J.C. Lachrymal determinations: methods and updates on biopharmaceutical and clinical applications. Ophthalmic Res. 36:183-194, 2004.

22. Sebbag, L., Harrington, D.M., and Mochel, J.P. Tear fluid collection in dogs and cats using ophthalmic sponges. Vet. Ophthalmol. 2017, 1-6.

23. Federici, T.J. The non-antibiotic properties of tetracyclines: clinical potential in ophthalmic disease. Pharmacol. Res. 64:614-623, 2011.

24. Wilson, R.C., Kemp, D.T., Kitzman, J.V., and Goetsch, D.D. Pharmacokinetics of doxycycline in dogs. Can. J. Vet. Res. 52:12-14, 1988.

25. López-Cisternas, J., Castillo-Díaz, J., Traipe-Castro, L., and López-Solís, R.O. Use of polyurethane minisponges to collect human tear fluid. Cornea. 25:312-318, 2006.

26. Fraunfelder, F.W., Solomon, J., and Mehelas, T.J. Ocular adverse effects associated with cyclooxygenase-2 inhibitors. Arch. Ophthalmol. 124:277-279, 2006.

27. Raizman, M.B., Hamrah, P., Holland, E.J., et al. Druginduced corneal epithelial changes. Surv. Ophthalmol. 62: 286-301, 2017.

28. Monaco, F., Mutani, R., Mastropaolo, C., and Tondi, M. Tears as the best practical indicator of the unbound fraction of an anticonvulsant drug. Epilepsia. 20:705-710, 1979.

29. Sato, S., Nakajima, M., Honda, A., Konishi, T., and Miyazaki, H. Pharmacokinetics of theophylline in Guinea pig tears. Drug Metab. Pharmacokinet. 22:169-177, 2007. 
30. Tan, A., Boudreau, N., and Lévesque, A. Internal standards for quantitative LC-MS bioanalysis. In: Xu, Q.A., and Madden, T.L., eds. LC-MS in Drug Bioanalysis. Boston, MA: Springer US; 2012; p. 1-32.

31. KuKanich, K., and KuKanich, B. The effect of sucralfate tablets vs. suspension on oral doxycycline absorption in dogs. J. Vet. Pharmacol. Ther. 38:169-173, 2015.

32. Tabbara, K.F., and Cooper, H. Minocycline levels in tears of patients with active trachoma. Arch. Ophthalmol. 107: 93-95, 1989.

33. Fullard, R.J., and Tucker, D.L. Changes in human tear protein levels with progressively increasing stimulus. Invest Ophthalmol. Vis. Sci. 32:2290-2301, 1991.

34. Hsu, H.H. Particle movement in paper porous media: influence factors and model (Master's thesis). 2010; pp. 97. Available at: https://macsphere.mcmaster.ca/bitstream/11375/ 9380/1/fulltext.pdf. Last accessed date: January 14, 2018.

35. Monk, C.S., Jeong, S.Y., Gibson, D.J., and Plummer, C.E. The presence of minocycline in the tear film of normal horses following oral administration and its anticollagenase activity. Vet. Ophthalmol. 2017. DOI: 10.1111/vop.12479.

36. Denisin, A.K., Karns, K., and Herr, A.E. Post-collection processing of Schirmer strip-collected human tear fluid impacts protein content. Analyst. 137:5088-5096, 2012.
37. Mishima, S., Gasset, A., Klyce, S.D., and Baum, J.L. Determination of tear volume and tear flow. Invest Ophthalmol. 5:264-276, 1966.

38. Arnold, D.R., Granvil, C.P., Ward, K.W., and Proksch, J.W. Quantitative determination of besifloxacin, a novel fluoroquinolone antimicrobial agent, in human tears by liquid chromatography-tandem mass spectrometry. J. Chromatogr. B Analyt. Technol. Biomed. Life Sci. 867:105-110, 2008.

Received: January 14, 2018

Accepted: March 12, 2018

Address correspondence to:

Dr. Lionel Sebbag

Department of Biomedical Sciences

Department of Veterinary Clinical Sciences Iowa State University

College of Veterinary Medicine 1809 S Riverside Drive

Ames, IA 50010

E-mail: 1sebbag@iastate.edu

Appendix A1. Number of Tear Samples in Dogs and Cats for Which Doxycycline Measurement Was Below Limit of Quantification, Detailing the Collection Device and Extraction Method Used as Well as the Associated Volume of Tears Absorbed

\begin{tabular}{|c|c|c|c|c|}
\hline & \multicolumn{2}{|c|}{ Schirmer strips } & \multicolumn{2}{|c|}{ PVA sponges } \\
\hline & Centrifugation & Methanol elution & Centrifugation & Methanol elution \\
\hline \multicolumn{5}{|l|}{ Dogs } \\
\hline Number of samples BLQ & $0 / 18$ & $3 / 18$ & $0 / 18$ & $4 / 18$ \\
\hline Mean volume absorbed $(\mu \mathrm{L})$ & 18.3 & 18.9 & 40.3 & 19.1 \\
\hline \multicolumn{5}{|l|}{ Cats } \\
\hline Number of samples BLQ & $2 / 18$ & $7 / 18$ & $3 / 18$ & $9 / 18$ \\
\hline Mean volume absorbed $(\mu \mathrm{L})$ & 16.1 & 16.6 & 26.0 & 18.1 \\
\hline
\end{tabular}

BLQ, below limit of quantification; PVA, polyvinyl acetal. 